\title{
Corela
}

Cognition, représentation, langage

HS-1 1 | 2012

RJC Cotexte, contexte, situation

\section{Italien et sarde dans l'espace urbain de Cagliari : contact de langues et contexte d'interaction}

\section{Giovanni Depau}

\section{(2) OpenEdition}

\section{Journals}

Édition électronique

URL : http://journals.openedition.org/corela/2179

DOI : $10.4000 /$ corela. 2179

ISSN : $1638-573 \mathrm{X}$

\section{Éditeur}

Cercle linguistique du Centre et de I'Ouest - CerLICO

\section{Référence électronique}

Giovanni Depau, «Italien et sarde dans l'espace urbain de Cagliari : contact de langues et contexte d'interaction », Corela [En ligne], HS-11 | 2012, mis en ligne le 02 avril 2012, consulté le 01 mai 2019. URL : http://journals.openedition.org/corela/2179; DOI : 10.4000/corela.2179

Ce document a été généré automatiquement le 1 mai 2019.

\section{(c) (i) (2)(2)}

Corela - cognition, représentation, langage est mis à disposition selon les termes de la licence Creative Commons Attribution - Pas d'Utilisation Commerciale - Partage dans les Mêmes Conditions 4.0 International. 


\title{
Italien et sarde dans l'espace urbain de Cagliari : contact de langues et contexte d'interaction
}

\author{
Giovanni Depau
}

\section{Considérations préliminaires}

1 La question du contexte en linguistique a été abordée dans plusieurs travaux sur la production verbale : il s'agit en effet d'une notion nécessaire à l'analyse des pratiques langagières, malgré son caractère problématique souligné à plusieurs reprises par les spécialistes du secteur, «au vu de sa plasticité et des multiples acceptions qu'elle est susceptible de recevoir $»^{1}$. Si le langage est une activité sociale complexe, la clé de son interprétation réside dans la prise en compte « active » de son rapport dynamique avec ses conditions de réalisation concrète.

2 Notre contribution vise à fournir des éléments de réflexion sur le rôle du contexte d'interaction dans un cadre de production bilingue. Cette étude se fonde sur l'analyse d'un corpus oral consistant d'un ensemble de textes bilingues «sarde-italien » recueillis (pour un total d'environ 16 heures d'enregistrements) dans des contextes interactionnels assez variés et se caractérisant par divers degrés de formalité 2 .

La Sardaigne constitue notre domaine d'enquête spécifique. L'italien s'avère la langue la plus répandue dans l'île (non seulement dans les situations plus formelles) à côté du sarde, qui demeure une langue minoritaire importante, voire, la plus répandue parmi les langues minoritaires de l'espace national italien ${ }^{3}$. Dans la pratique langagière quotidienne, le contact des codes est récurrent. Plus particulièrement, à Cagliari, chef lieu régional et notre lieu d'observation, l'impact de l'italien s'exerce, sur le plan fonctionnel, à travers l'emploi diffusé de ce dernier au détriment du sarde; en outre, le dialecte cagliaritain est considéré par les spécialistes du sarde comme très innovateur au niveau structurel, avec une forte tendance à l'italianisation ${ }^{4}$. 


\section{Approche interprétative et phénomènes de code-switching}

4 L'analyse effectuée dans notre étude est centrée principalement sur une démarche interprétative fondée sur l'observation du code-switching à l'intérieur du contexte discursif où il se réalise. La perspective interactionnelle permet d'appréhender l'importance pragmatique et le contenu communicationnel de la commutation codique ${ }^{5}$, à travers l'estimation de ses fonctions spécifiques à l'intérieur des épisodes conversationnels analysés individuellement.

5 Selon cette approche, la plupart des conversations permettent un choix très large du point de vue des comportements langagiers ${ }^{6}$. Le code-switching est lié à la dynamique de la conversation et sa réalisation n'est pas totalement prévisible à travers une démarche déterministe le considérant comme une variable dépendante de paramètres sociologiques classiques (âges, sexe, degré de scolarisation, classe sociale, etc.), ou de facteurs diaphasiques comme le sujet et les relations de rôle entre les interactants. La pertinence du statut des locuteurs est liée elle-même au déroulement de l'interaction (cf. Dittmar, 2005). Ces statuts sont certes associés à l'exercice légitime de rôles interactionnels. Toutefois, au-delà du statut social de chaque partenaire, la relation entre les interactants est le résultat d'une négociation entre ces partenaires, qui se crée, renforce et renouvelle dans le cadre contextuel de l'échange. Cette convention pouvant varier au long de l'échange verbal, les positions respectives de chaque locuteur peuvent subir elles aussi des modifications. Ainsi, dans le cadre de la « gestion » de l'interaction, un comportement linguistique adapté au contexte discursif et à son (ses) interlocuteur(s) s'avère nécessaire pour atteindre l'accomplissement correct de l'échange.

6 Les facteurs déclenchant la commutation - comme, par exemple, un changement de sujet ou d'interlocuteur - ne doivent donc pas être vus comme de véritables « causes », car ils ne l'occasionnent pas nécessairement. Le jeu d'interprétation que les participants opèrent continuellement à l'intérieur de l'interaction se fait à travers l'utilisation des ressources plurisémiotiques disponibles. Cela rend l'interaction à la fois le résultat et le lieu d'élaboration du contexte.

7 Autrement dit, le code-switching, loin d'être unilatéralement influencé ou préétabli par la situation, se configure plutôt comme un des « outils » contribuant à une réinterprétation et redéfinition constante de celle-ci, dans un processus dynamique et bidirectionnel entre interactants, se réalisant à travers une activité de contextualisation de l'échange permettant à chacun des participants d'aller au-delà du niveau locutoire du message. Le code-switching joue ainsi une fonction de contextualisation cue (comprenant les signes non verbaux accomplis par des gestes, des regards, des mouvements du corps, la prononciation, le rythme, etc. ; cf. Gumperz, 1982 ; cf. aussi Auer, 1992 ; Álvarez Cáccamo, $1990)$ et, tout comme les autres indices de contextualisation, reflète et présuppose des conventions apprises à travers les pratiques interactionnelles des locuteurs d'une communauté. Il fournit une série d'indications permettant de 'catégoriser' les locuteurs et de saisir des informations concernant leur répertoire verbal et leur comportement linguistique. C'est une ressource communicationnelle supplémentaire à disposition des locuteurs bilingues lorsqu'ils se trouvent dans des situations où ils peuvent mobiliser la totalité de leur répertoire verbal. De ce point de vue, le passage de code doit être considéré comme une possibilité ouverte au libre choix du locuteur de l'utiliser ou bien 
l'ignorer pour atteindre des buts interactionnels déterminés, ou pour résoudre des problèmes liés à la conduite et à l'organisation de l'activité conversationnelle.

Notre analyse se réalise, sur le plan méthodologique et terminologique, dans le cadre de la répartition entre le code-switching lié aux participants et le code-switching lié au discours, suivant une tradition de recherche désormais consolidée à partir, notamment, des répartitions élaborées par Auer (1984) ${ }^{7}$ et particulièrement enracinée dans le domaine italo-roman. En effet, les recherches menées sur ce terrain témoignent de la productivité de l'approche pragmatique et confirment le fait que certains loci conversationnels (Auer, 1995) sont particulièrement sensibles au changement de code, au-delà du système grammatical spécifique impliqué et du contexte spatial et social où la conversation se produit ${ }^{8}$.

9 Un cas exemplaire d'usage du code-switching dans le développement de la relation conversationnelle pour des buts pragmatiques est l'expression de la convergence / divergence, permettant aux interactants de manifester leur proximité, leur accord, ou bien au contraire, leur éloignement, voire, leur désaccord à l'égard de son interlocuteur. Il s'agit, ainsi, d'une forme de réalisation de la préférence linguistique, très productive dans l'interprétation des phénomènes de commutation. Il faut préciser que le concept de "code-switching" fait référence au passage d'un code à l'autre dans la production langagière d'un même locuteur à l'intérieur du même événement discursif. À la rigueur, donc, dans l'analyse des phénomènes de préférence nous n'avons pas affaire à des phénomènes de commutation codique strictu sensu, mais plutôt, comme le soulignent Auer (1984) et Berruto (1990), à des stratégies ${ }^{9}$ de choix codique de la part de chaque locuteur, d'accommodation ou de dissociation par rapport à la production verbale de son partenaire conversationnel. Cependant, cette catégorie de phénomènes s'avère très importante du point de vue interactionnel et requiert une analyse de type interprétatif.

\section{Analyse des interactions}

Les extraits suivants nous permettront de voir comment le contraste entre l'usage et le contexte peut provoquer des conflits linguistiques mettant en évidence la " confrontation" pour le contrôle de la situation interactionnelle et, par conséquent, quels dispositifs verbaux et plus généralement communicationnels sont mis en place pour résoudre ces conflits.

11 Les échanges présentés se caractérisent par l'utilisation de l'italien comme langue principale, à laquelle s'ajoutent des insertions en sarde. Dans ce cadre, la fonction de wecode attribuée au sarde est mise en valeur justement par la présence extrêmement minoritaire de la langue locale à l'intérieur des conversations et par son emploi juxtaposé à celui de l'italien.

\section{Setting et rôle des interactants : tentatives de convergences à l'ASL, pt 1}

Comme nous l'avons souligné auparavant, la valeur attribuée aux langues par les interactants peut être liée au contexte d'énonciation et aux inputs provenant des tours de parole précédents. L'opposition entre convergence et divergence recouvre ainsi également la dichotomie we-code/they-code. Celle-ci est liée à l'attribution d'une valeur 
identitaire aux codes en présence et, comme le montrent plusieurs études, dépasse le champ d'un véritable contraste "ethno-linguistique» opposant le code d'un groupe minoritaire de locuteurs à langue officielle du groupe dominant pour indiquer, plutôt, une forme d'implication personnelle s'appliquant à des situations liées aux expériences " partagées par les membres du groupe » versus « externes à la réalité du groupe ${ }^{10}$ dans le cadre limité de l'interaction verbale.

C'est le cas d'un échange se produisant dans un bureau de l'office régional de la santé publique ( ASL » : Azienda Sanitaria Locale). La conversation se déroule autour de trois participants : l'employé ; une fille de 26 ans qui se renseigne sur les services auxquels elle a droit en cas de voyage à l'étranger; la mère de cette dernière. Il s'agit d'une conversation en principe asymétrique et dont le degré de formalité serait, sinon élevé au moins neutre ${ }^{11}$.

Extrait $1 \rightarrow$ Bureau ASL (Azienda Sanitaria Locale « Agence régionale pour la santé publique "; matin. Employé (EH50), F26 (jeune femme, usager du service d'information), MèreF26.

1 F26 quindi io per esempio mi rompo una gamba, cosa faccio?

2 EH50 <INC> ascolti vada in chiesa perché per farci stare <INC> boh! ((2s))

pensendu a si segai una gamba!

3 F26 il rimborso me lo danno qui o lì ?

$4 \mathrm{EH} 50$ no, lei conservi le fatture / dove deve andare?

5 F26 in Belgio, a Bruxelles

6 EH50 questo è il foglio che le permette assistenza per trenta giorni, poi quando le arriverà la tessera sanitaria la sua cara mamma gliela spedisce all'estero

7 F26 e ma il rimborso lo chiedo in questo ufficio poi [<inc>]

8 EH50 ((se moque d'elle, la traite comme si elle était stupide)) [eja eja è] già la terza volta che glielo sto dicendo $[<\mathrm{INC}>]=$

9 F26 [io vengo da] lei / lei mi da i soldi ? =

= (8) REMBORSEMENT ((SIC)) PAS! ((2 s)) DEMANDER C'EST ICI! OK ?! [GLIELO

DICO $=$

$=(9)$ [grazie !] ((elle et sa mère se lèvent et se dirigent vers la sortie))

= (8) IN FRANCESE IN BELGO ((sic)) E IN COSO / ANCHE IN UCRAìNO SE VUOLE

((2 s)) A SI BIRI!

10 Mère ((près de la porte, en train de sortir)) a si biri / meglio in sardo !

1 F26 Donc moi par exemple je me casse une jambe, qu'est-ce que je fais?

2 EH50 <INC> écoutez allez à l'église parce que pour y faire ((rester / rentrer) $)<\mathrm{INC}>$

boh! ((2 s)) elle pense à se casser une jambe!

3 F26 Le remboursement on me le donne ici ou là-bas?

4 EH50 Non, conservez les factures / où est-ce que vous devez aller?

5 F26 En Belgique, à Bruxelles

6 EH50 Ceci est le papier qui vous permet l'assistance pendant trente jours, après quand la carte d'assurance maladie vous parviendra votre chère maman vous l'envoie à l'étranger

7 F26 Mais le remboursement je le demande dans ce bureau après [ $<I N C>]$

8 EH50 ((se moque d'elle, la traite comme si elle était stupide)) [OUAIS, OUAIS, $\left.C^{\prime} \mathrm{EST}\right]$ déjà la troisième fois que je vous le dis $[<\mathrm{INC}>]=$

9 F26 [Je viens chez] vous/vous me donnez l'argent ? =

= (8) REMBORSEMENT ((SIC)) PAS ! ((2 s)) DEMANDER C'EST ICI ! OK ? ! [JE VOUS LE DIS] $=$

$=(9)$ [Merci !] ((elle et Mère se lèvent et vont vers la sortie))

= (8) EN FRANÇAIS EN BELGO ((sic)) ET EN MACHIN / MÊME EN UKRAINIEN SI VOUS VOULEZ ((2 s)) AU REVOIR!

10 Mère ((près de la porte, en train de sortir)) au revoir / c'est mieux en sarde! 
En observant l'échange dans son ensemble, nous remarquons que l'employé s'exprime de façon de plus en plus informelle : dans le $\mathrm{t} 2$ il répond en sarde à une question que F26 lui avait posée à l'aide d'un exemple quelque peu " extrême"; dans ce premier tour de dialogue le ton reste cordial.

L'emploi du sarde comme élément de clôture définitive, voire, brutale, de la conversation, constitue un élément-clé de l'échange. Quand, dans le t8, EH50 réagit de manière moqueuse aux interpellations de la fille, il utilise aussi le français - qui a ici une fonction mimétique, car F26 doit partir en Belgique - et produit un discours en quelque sorte métalinguistique qui s'achève dans le t8 par la formule de salutation en sarde « a si biri $»^{12}$. Dans cette perspective la commutation a une fonction assez évidente d'interruption définitive, liée à une expression d'emphase (cf. Sacks, Schegloff, Jefferson 1974 ; Schegloff, Sacks 1973). L'aspect le plus intéressant est l'adhésion explicite de la part de la mère (t10) à la forme sarde «a si biri »: en effet, elle avait gardé jusque-là un rôle absolument marginal dans l'interaction. Dans sa réponse, la convergence se produit non seulement au niveau strictement codique, mais aussi et surtout «idéologique ", car le sarde se voit attribué une marque très forte - mais indubitablement limitée à la surface - de we-code, comme l'est explicité par le commentaire méta- épilinguistique portant sur le choix du sarde au sein d'une interaction ("meglio in sardo»), qui se fait pourtant en italien. Cela engendre une sorte de croisement et de contraste de cues concernant le contenu interactionnel de ce tour de parole: d'une part, maintien d'une distance formelle, de l'autre, expression d'une connivence « héritée » des tours précédents, qu'il ne serait pas possible d'appréhender sans tenir compte de la succession des tours antécédents.

Cet exemple illustre l'usage du sarde comme we-code ne véhiculant pas de réelle intention de revendication identitaire: l'affirmation est hyper-contextualisée au niveau de l'échange et strictement liée aux tours de parole précédents, en particulier, est finalisée à la clôture de la conversation. Ainsi, il y a une volonté " conciliatrice " s'exprimant à travers une convergence linguistique avec l'employé, plutôt qu'une véritable revendication linguistico-identitaire indépendante du contexte énonciatif.

\section{Setting et rôle des interactants : tentatives de convergences à l'ASL, pt 2}

La forte contextualisation de l'échange précédant peut trouver une confirmation dans une comparaison avec une autre situation communicative, où une tentative de converger vers le sarde en tant que we-code aboutit à l'échec.

Le setting est encore le bureau de l'ASL : l'interaction en question s'est déroulée juste après celle que nous venons de décrire dans l'extrait 1.

Les participants sont au nombre de deux : G et l'employé de l'ASL, EH50. La discussion porte sur l'attribution à $G$ de la carte européenne d'assurance maladie. Ses données personnelles n'ont pas été enregistrées dans les archives électroniques de l'agence, comme l'explique EH50 avec un ton de sarcasme envers les responsables institutionnels de la Région Sardaigne.

Ensuite, les deux interlocuteurs parlent de la renonciation au service du médecin généraliste que $G$ avait opérée lors de son premier déplacement en France, et du fait qu'à son retour en Italie, dans sa ville d'origine, il n'avait pas « récupéré » son droit au médecin traitant. 


\section{Extrait $2 \rightarrow$ Bureau ASL (Azienda Sanitaria Locale «Agence régionale pour la santé publique ») ; matin. Employé (EH50), G (Giovanni).}

$1 \mathrm{EH} 50$ anche lei è tra quel $2030 \%$ che l'assessore alla sanità la dirindina ${ }^{13}$ insieme con il nostro illuminato non le ha inserito i dati sanitari

$2 \mathrm{G}$ eh, si 'nd'è scarescia!

3 EH50 siccome sono due stranieri che le / chi è il suo medico ?

$4 \mathrm{G} \mathrm{XYZ} \mathrm{però} \mathrm{è} \mathrm{vera} \mathrm{una} \mathrm{cosa} \mathrm{io} \mathrm{sono} \mathrm{partito} \mathrm{in} \mathrm{Francia,} \mathrm{io} \mathrm{viv/} \mathrm{eh} \mathrm{vivo} \mathrm{in} \mathrm{Francia}$ per cui ho la residenza qua a Cagliari però ho fatto il / avevo rinunciato al medico di qua per cui volevo [sapere cosa] / ah

5 EH50 [non fa niente] non fa niente / io al limite le posso anche / non ce l'ha il medico adesso?

$6 \mathrm{G} \mathrm{no}:$ /ah, forse eh/allora/

7 EH50 se non lo ha ripreso non ce l'ha eh

$8 \mathrm{G}$ no allora non ce l'ho / era la dottoressa XYZ era

$9 \mathrm{EH} 50$ e intzàndusu non du denir ${ }^{i}$ / non fa niente non lo mettiamo gli mettiamo solo quattro fesserie qua di inizio assistenza come dati sanitari tanto lo prende / ecco qua, le abbiamo programmato la tessera sanitaria è a posto

1 EH50 Vous êtes aussi parmi ces $2030 \%$ de personnes dont l'adjoint chargé à la Santé 'la dirindina' avec notre Éclairé n'a pas fourni les données concernant votre fiche sanitaire

$2 \mathrm{G}$ Eh, elle l'a oublié!

3 EH50 Comme ce sont deux étrangers qui ((vous / les))/ qui est votre médecin?

$4 \mathrm{G} \mathrm{XYZ} \mathrm{toutefois} \mathrm{c'est} \mathrm{vrai} \mathrm{que} \mathrm{je} \mathrm{suis} \mathrm{parti} \mathrm{en} \mathrm{France} \mathrm{j'habit/} \mathrm{eh} \mathrm{habite} \mathrm{en} \mathrm{France}$ donc j'ai la résidence ici mais j'ai fait le / j'avais renoncé au médecin traitant donc je voulais [savoir ce que] / ah

5 EH50 [c'est pas grave] c'est pas grave / moi à la limite je pourrais même/vous n'avez pas de médecin traitant maintenant?

$6 \mathrm{G}$ No : /ah, peut-être eh/alors/

7 EH50 Si vous ne l'avez pas repris vous ne l'avez pas eh

$8 \mathrm{G}$ Non alors je ne l'ai pas/c'était Madame le Docteur XYZ c'était

9 EH50 Et alors vous ne l'avez pas / ce n'est pas grave on ne le met pas ((dans la fiche sanitaire)) on y met seulement quatre informations sans valeur ici pour le début d'assistance comme données sanitaires, de toute façon c'est suffisant / voilà, on vous a programmé la carte sanitaire c'est tout bon

Notons une sorte d'évolution dans le comportement de l'employé au long de la conversation. En effet, EH50 tient un comportement très amical vis-à-vis de G, qui compte tenu aussi du fait que le même employé s'était exprimé en sarde dans la conversation précédente avec F26 et sa mère - commente en sarde l'information reçue (t2 : «eh, si 'nd'è scarescia!»). La tentative de solliciter implicitement l'employé à utiliser le sarde n'aboutit pas à un résultat positif, car ce dernier continue à s'exprimer en italien ; la conversation se déroule dans la langue nationale jusqu'au t9, qui s'ouvre avec un énoncé en sarde (" e intzàndusu non du deniri ») par EH50 avant de revenir à l'italien avec un propos d'encouragement et de minimisation des difficultés. L'énoncé en sarde prononcé par l'employé consiste aussi en une réitération de celui produit en italien par $\mathrm{G}$ dans le tour de parole précédent (« no allora non ce l'ho / [...] »).

Dans ce cas, donc, la convergence manquée sur le plan linguistique en occasion du premier passage au sarde de $G$ dans le t2 se produit ensuite et coïncide avec une expression de solidarité personnelle. Le choix d'EH50 de passer immédiatement à l'italien montre qu'il n'y a pas de véritable volonté de sa part de converser en sarde et que l'usage de cette langue a plutôt une fonction de mise au point de la relation interactionnelle.

Il nous paraît aussi que le choix opéré par EH50 de ne pas donner suite du point de vue linguistique à l'énoncé en sarde produit par $G$ dans le t2 est motivé par une sorte 
d'imposition «tacite» de la part de l'employé du caractère asymétrique de la conversation: l'employé bénéficierait de par son statut du droit exclusif de gérer l'échange verbal et, par conséquent, de choisir la clé de l'interaction et éventuellement le code qui lui est associé. Il marque d'abord sa distance vis-à-vis de G (refus implicite de converger vers le sarde, qui aurait peut-être conduit à une baisse de registre ou bien à des moments de manque de clarté des rapports réciproques), ensuite il montre une nouvelle attitude (usage du sarde avec reprise de l'énoncé précédent) pour marquer, lui, le rapprochement.

\section{Observations sur un cas de négociation conversationnelle}

Les deux premiers exemples évoqués illustrent la manière dont la relation verbale entre les interlocuteurs est négociée dans le développement de l'interaction, suivant l'organisation séquentielle de celle-ci. L'activité de négociation ne concerne pas seulement la sphère linguistique mais aussi la parité respective des partenaires et donc la gestion de l'échange dans sa globalité.

Une dernière interaction, se déroulant dans un bar - bureau de tabac entre deux jeunes d'environ vingt ans (Ale, le caissier et Christian, un client), présente l'influence probante des aspects pragmatiques concernant les choix de langues dans l'organisation et l'évolution de l'interaction.

Dans cette conversation, l'attribution de différentes valeurs sémantico-discursives selon la langue utilisée (sarde ou italien) caractérise le même élément lexical: la forme équivalente à « dépêche-toi ». Plus précisément, une valeur non marquée est attribuée par les deux interactants, dans cette interaction, à la forme italienne «muoviti», alors que la forme sarde "movirì est connotée pragmatiquement et est associée à une intention de provocation.

Extrait $3 \rightarrow$ Movirì vs Muoviti ; Bar - bureau de tabac, matin. Ale. (caissier), Ch. (client) : deux jeunes d'environ 20 ans. Autres participants : autres clients faisant la queue et qui n'interviennent pas (dont $G$ ).

1 Ale ciao Roby / dimmi Christian

2 Ch due caffè e un « Settemmezzo »

3 Ale due caffè $((2 \mathrm{~s}))$ ?

4 Ch e un « Settemmezzo » / moviri

5 Ale adesso tu $\mathbf{a} \Sigma$ pett $/<\mathrm{INC}>$

$6 \mathrm{Ch}$ da' ajo' oh/

7 Ale $((=>C h))$ da' $:$ vien/ (( $3^{\text {ème }}$ personne réthorique ; interlocuteur fictif $\left.)\right)$ che stava giocando/ $((\Rightarrow \mathrm{Ch}))$ minimo $<\mathrm{mi}>$ devi chiedere $\Sigma$ cusa/ ((au client suivant, gentiment)) prego

8 Ch da': me li dai i caffè :

9 Ale sh : mo' ti faccio aspettare di nuovo/ cazzi tuoi

10 Ch dai Ale, devo <INC> / muoviti

11 Ale chiedimi scusa!

$12 \mathrm{Ch}$ a : 'oghendimi scusa!

13 Ale e allora non ti servo!

14 Ch scusa dai muoviti ! ((petit rire))

15 Ale visto : ? bravo !

$16 \mathrm{Ch}$ e i due caffé da portar via/ e movirì ancora ((petit rire)) ((Ale arrête de

s'occuper de $\mathrm{Ch}))((2 \mathrm{~s}))$ da' ajò

17 Ale $((=>$ autre client $))$ prego !

18 Ch me li dai ((2 s)) i caffè me li dai ? ((4 s)) dai muoviti ! 
19 Ale chiedimi scusa!

1 Ale Ciao Roby / dis-moi Christian

2 Ch Deux cafés et un ticket du loto

3 Ale Deux cafés ((2s))?

4 Ch Et un ticket du loto / dépêche-toi

5 Ale Là, tu attends/ <INC>

$6 \mathrm{Ch}$ Allez allez oh/

7 Ale A :llez vien/ (( $3^{\text {ème }}$ personne rhétorique; interlocuteur fictif $\left.)\right)$ qu'il voulait jouer / ( $=>\mathrm{Ch})$ ) il faut minimum que tu t'excuses / ((au client suivant, gentiment))

oui, s'il vous plaît

8 Ch Alle :z tu me les donnes les café :s

9 Ale Ch :ut (( = tais-toi !)) là je te fais attendre encore/ tant pis pour toi

$10 \mathrm{Ch}$ Allez Ale, je dois $<\mathrm{INC}>$ / dépêche-toi

11 Ale Excuse-toi !

$12 \mathrm{Ch}$ Ah il me sort plein d'excuses!

13 Ale Alors je te sers pas!

14 Ch Désolé allez dépêche-toi ! ((petit rire))

15 Ale T'as vu :? ! Bon mec!

16 Ch Et les deux cafés à emporter/et dépêche-toi encore ((petit rire)) ((2 s)) Allez

allez

17 Ale (( => autre client)) S'il vous plait !

$18 \mathrm{Ch}$ Tu me les donnes? ((2 s)) Les cafés, tu me les donnes? ((4 s)) Allez dépêche-

toi

19 Ale Excuse-toi !

dont plusieurs habituels de l'endroit. Le caissier [Ale], interagit avec les consommateurs, il est très à l'aise et rapide dans son travail, et dans le t1 s'adresse à son dernier client en l'appelant par un diminutif de son prénom. Pour le règlement des consommations les clients se positionnent en face d'Ale, alors que celui avec qui aura lieu l'interaction [Ch] se trouve débout à sa droite; il ne changera pas de position jusqu'à la fin de l'échange. On voit qu'ils se connaissent, car dans le $t 1$, après avoir servi un autre client, Ale s'adresse directement à Ch en l'appelant par son prénom (Christian) et ensuite ce dernier appellera le caissier "Ale ", diminutif d'Alessio ou Alessandro. Il s'agit donc d'une interaction à la fois symétrique - car les deux locuteurs ont à peu près le même âge, se connaissent, se tutoient - et asymétrique, puisqu'ils se trouvent dans un contexte public, de transaction commerciale où les deux interlocuteurs jouent deux rôles différents (vendeur / client), et en présence de personnes étrangères.

Un motif majeur d'intérêt de cette conversation repose sur le contraste qui se crée entre les interprétations - surtout initiales - que chacun des deux participants principaux donne à l'interaction et à l'ensemble situationnel où celle-ci a lieu : en effet, Ch s'oriente tout de suite et de manière manifeste vers une relation plutôt symétrique, tandis qu'Ale réagit à cette attitude en s'orientant plutôt vers une relation asymétrique, où lui-même serait dans une condition de supériorité vis-à-vis de son interlocuteur. On remarque, en effet, que le contrôle de l'échange dans son ensemble est tenu par Ale.

Le dissentiment entre les deux interactants est engendré par la réponse de Ch lorsqu'Ale (t3) montre une hésitation, n'ayant pas saisi toute la commande de son jeune client qui s'adressait à lui pour recevoir et régler deux cafés à emporter et un billet du loto. En effet, dans le $\mathrm{t} 4 \mathrm{Ch}$ répète " $\mathrm{e}$ un 'Settemmezzo'", mais ajoute ce que nous pouvons considérer une première provocation à l'égard d'Ale, avec l'injonction en sarde "movirì " (littéralement, « bouge-toi »). Ale réagit donc en ignorant $\mathrm{Ch}$ et sa commande, et s'adresse 
à un autre client situé devant lui. Il est alors possible d'entendre l'énoncé " adesso tu a pett/ <INC>» («maintenant tu attends») se caractérisant aussi du point de vue linguistique par la prépalatalisation de la fricative [s] suivie d'occlusive (italien standard « aspetti »). La comparaison avec d'autres occurrences enregistrées dans ce même corpus montre que cette réalisation phonétique se situe à un degré diastratique et diaphasique bas, informel de l'italien parlé à Cagliari. Cette réplique confirme que la provocation lancée par $\mathrm{Ch}$ a effectivement atteint son interlocuteur et nous pouvons, ainsi, interpréter ce passage comme le début du conflit. En effet Ch ( $t 6:$ : da' ajo' oh/») est visiblement mal à l'aise à cause de la réaction inattendue d'Ale : son refus ferme de servir le jeune client change le cadre relationnel avec celui-ci, en affaiblit de manière évidente la position en public et le ridiculise, d'autant plus qu'à l'origine de cette situation se trouve, justement, son attitude provocatrice.

La trêve accordée par Ale à Ch au début du $\mathrm{t} 7$ ( $(\mathrm{da}$ ' : vien/ che stava giocando/») est seulement apparente, comme l'indique le changement de ton adopté par le caissier dans le même tour de parole («[...] chiedere $\Sigma$ cusa »). Le pardon public est en réalité accordé avec condescendance, de manière presque ironique. Au contraire, lorsqu'il impose à $\mathrm{Ch}$ de s'excuser publiquement, il s'exprime d'un ton péremptoire auquel vient s'ajouter la réalisation prépalatale de la fricative devant consonne occlusive ( $\Sigma$ cusa ») témoignant $\mathrm{du}$ fait que pour Ale le conflit est encore en cours. Ensuite, sans attendre la réponse de $\mathrm{Ch}$, le caissier sert un autre client et continue à ignorer son interlocuteur principal, insoucieux de son insistance dans le t8. Ainsi faisant, il vise à marquer davantage sa supériorité face à Ch et en sanctionner l'excès de braverie.

31 Le conflit devient plus explicite dans le t9 avec la réaction d'Ale à l'insistance de Ch: en effet, il impose le silence à son interlocuteur à travers le signal «sh:» («chut») accompagné par la déclaration « mo' ti faccio a $\Sigma$ pettare di nuovo » « là je te fais attendre encore" et par l'expression grossière "cazzi tuoi » qui achève son tour de parole. Concernant les choix lexicaux, signalons l'emploi de l'adverbe «mo" » (" maintenant ») variante diatopique (< sarde immòi) et diaphasique ( + informelle), des autres adverbes italiens adesso et ora $^{14}$. Au niveau phonétique notons encore une fois la réalisation prépalatale de $[\mathrm{s}]$ dans «a $\mathrm{a}$ pettare» («attendre »). Dans les deux cas, il émerge une tendance au passage vers un registre linguistique plus bas coïncidant avec le discours adressé par Ale vers $\mathrm{Ch}$, tandis que quand le caissier s'adresse aux autres clients le registre demeure neutre, peut-être relativement informel - comme lorsque, dans le t1, il dit «ciao " à son client Roby avant de s'adresser pour la première fois à $\mathrm{Ch}$ - mais totalement dépourvu de l'agressivité ou la vulgarité que l'on retrouve, au contraire, dans les échanges avec $\mathrm{Ch}$.

Dans le tour t10, un changement intervient dans l'interaction: en effet, l'impératif « dépêche-toi » qui est à l'origine du conflit entre les deux locuteurs, est utilisé davantage par Ch; cette fois, cependant, le client s'exprime en italien (« muoviti»). Ce changement, qui n'entraîne pas de réaction particulière de la part d'Ale, peut être motivé par le fait que maintenant le ton de la conversation devient de plus en plus aigre. En effet, bien qu'ils ne soient pas en train de se disputer, les deux interlocuteurs se trouvent dans une situation évidente de tension et d'imposition de sa propre personnalité face à l'autre. Le choix codique dans ce contexte précis peut donc être très relevant sur le plan pragmatique, même s'il concerne dans ce cas un seul élément lexical : Ch évite l'emploi du sarde - associé à la provocation du conflit - et avec le passage à l'italien se met dans une 
attitude que l'on pourrait définir de «non-plaisanterie » et n'accepte plus de subir ce traitement punitif de la part d'Ale.

Le jeune client reprendra en partie cette attitude de plaisanterie dans le t14, lorsqu'il décide de s'excuser face à Ale mais produit en même temps une nouvelle occurrence de "dépêche-toi » (en italien) : "scusa dai muoviti!». Ce nouveau "muoviti », qui est en plus accompagné par un léger rire, lui permettrait ainsi d'atténuer le poids de son acte d'excuses publiques; son comportement serait donc paraphrasable de la façon suivante: «je m'excuse mais je continue à te taquiner». Soulignons que ces deux occurrence italienne de " dépêche-toi » (t10 et t14) ne provoquent pas chez Ale la même réaction que celle en sarde : notamment, il se concentre plutôt sur l'acte de demande de pardon que sur l'acte d'imposition présent dans le même tour de parole. En effet, à partir du t15 Ale accepte les excuses rendues publiquement par $\mathrm{Ch}$ et met fin au conflit avec un ton de «victoire», exemplifié par "visto?! bravo!», qui peut se lire comme un acte d'apaisement.

Le t16 coïncide avec la reprise de confiance du jeune client qui essaie à nouveau d'obtenir, avec insistance, les deux cafés à emporter. Ch joue alors une autre fois la carte de la provocation avec l'élément linguistique « movirì » et provoque pour la deuxième fois une réaction négative chez Ale, qui se tourne vers un autre client. $\mathrm{Ch}$, ne voulant perdre la face publiquement, essaie immédiatement de changer la clé conversationnelle et de tempérer la réaction d'Ale ${ }^{15}$. Ainsi faisant, Ch cherche à mettre en relief le caractère de plaisanterie de sa provocation et donc adresse à son interlocuteur une invitation implicite à ne pas retourner sur le terrain de l'interaction conflictuelle.

Dans t18 et t19 l'alternance sarde-italien assume définitivement une configuration « régulière », et Ch utilise l'italien pour insister avec une attitude sérieuse vis-à-vis d'Ale (t18: « dai muoviti !»). De façon parallèle, la réaction d'Ale dans t19 (« excuse-toi ! ») fait encore référence à la forme en sarde du t16, alors qu'il ne semble pas intéressé à la forme italienne du t18, bien que celle-ci soit effectivement la dernière à avoir été prononcée.

\section{Une répartition en micro-contextes interactionnels}

Le rapport entre Ale et Ch se renouvelle à chaque phase de l'échange, laissant émerger la construction de micro-situations interactionnelles.

On remarque ainsi une symétrie entre, d'une part, l'alternance « attitude de provocation (Ch) versus réaction (Ale) » et, d'autre part, l'alternance « movirì vs muoviti », c'est-à-dire, l'emploi de l'élément « dépêche-toi » en sarde ou en italien.

La cooccurrence de ces deux différentes alternances peut être illustrée à l'aide du tableau qui suit, juxtaposant les comportements des deux locuteurs et mettant en évidence la présence de micro-contextes interactionnels. Ce tableau schématise les cinq différentes phases dans lesquels l'échange conflictuel entre Ale et $\mathrm{Ch}$ se déroule, et les éléments réguliers qui se manifestent à travers ces passages. Pour chaque phase, il est possible de mettre en relation cinq facteurs distinctifs: 1) l'attitude de départ de $\mathrm{Ch}$;2) son correspondant linguistique, se réalisant dans l'alternance entre les éléments lexicaux movirì et «muoviti »; 3) la perception, d'un point de vue général, d'Ale du comportement linguistique de $\mathrm{Ch} ; 4)$ le comportement d'Ale en réaction au comportement linguistique de $\mathrm{Ch} ; 5)$ le correspondant linguistique de la réaction d'Ale au comportement linguistique de Ch. 


\begin{tabular}{|c|c|c|c|c|c|}
\hline & \multicolumn{2}{|l|}{ CH (CLIENT) } & \multicolumn{3}{|l|}{ AL (CAISSIER) } \\
\hline & $\begin{array}{l}\text { Attitude } \\
\text { de départ }\end{array}$ & $\begin{array}{l}\text { Alternance } \\
\text { Movirì /Muoviti }\end{array}$ & $\begin{array}{l}\text { Perception } \\
\text { générale de } \\
\text { l'input }\end{array}$ & $\begin{array}{l}\text { Réaction : } \\
\text { comportement } \\
\text { vis-à-vis de Ch }\end{array}$ & $\begin{array}{l}\text { Réaction } \\
\text { linguistique }\end{array}$ \\
\hline 1 & $\begin{array}{l}\text { Détendue. } \\
\text { Provocation. } \\
\text { Plaisanterie }\end{array}$ & $\begin{array}{lcr}(\mathrm{t} 4) & \mathrm{E} & \text { un } \\
\text { Settemmezzo } & / \text { movirì }\end{array}$ & \begin{tabular}{|l} 
Négative \\
Il se sent \\
« insulté ». \\
Il ressent \\
movirì comme \\
une \\
provocation.
\end{tabular} & $\begin{array}{l}\text { Il ne le sert pas. } \\
\text { Il s'adresse à un } \\
\text { autre client. }\end{array}$ & $\begin{array}{l}\text { (t5) Adesso tu a } \\
\Sigma \text { pett/ <INC> } \\
\text { (t7) devi } \\
\text { chiedere } \Sigma \text { cusa } \\
\text { (t9) a } \Sigma \text { pettare }\end{array}$ \\
\hline 2 & $\begin{array}{l}\text { Sérieuse, } \\
\text { fin de la } \\
\text { plaisanterie }\end{array}$ & $\begin{array}{l}\text { (t10) Dai Ale, devo } \\
\text { <INC> / muoviti }\end{array}$ & $\begin{array}{l}\text { Neutre } \\
\text { Muoviti est } \\
\text { neutre : il n'y } \\
\text { a pas de } \\
\text { réaction } \\
\text { particulière à } \\
\text { cet élément. }\end{array}$ & $\begin{array}{l}\text { Il insiste envers } \\
\text { Ch afin qu'il } \\
\text { s'excuse. } \\
\text { Il sert un autre } \\
\text { client. }\end{array}$ & $\begin{array}{l}\text { (t11) Chiedimi } \Sigma \\
\text { cusa! }\end{array}$ \\
\hline 3 & $\begin{array}{l}\text { Intermédiaire, } \\
\text { atténuation du } \\
\text { poids de l'acte } \\
\text { s'excuser }\end{array}$ & $\begin{array}{l}(\mathrm{t} 14) \quad \text { Scusa dai } \\
\text { muoviti! ((petit rire) })\end{array}$ & $\begin{array}{l}\text { Indifférente } \\
\text { Muoviti est } \\
\text { ignoré. } \\
\text { L'aspect } \\
\text { important est } \\
\text { que Ch s'est } \\
\text { excusé. }\end{array}$ & $\begin{array}{ll}\text { Victoire, } & \\
\text { satisfaction. } & \\
\text { Il accepte les } & \text { les } \\
\text { excuses avec un } \\
\text { ton } & \text { de } \\
\text { supériorité. } & \end{array}$ & $\begin{array}{l}\text { (t15) Visto : ?! } \\
\text { Bravo! }\end{array}$ \\
\hline 4 & $\begin{array}{l}\text { Détendue. } \\
\text { Provocation } \\
\text { Plaisanterie }\end{array}$ & $\begin{array}{l}\text { (t16) E i due caffé da } \\
\text { portar via/ e movirì } \\
\text { ancora ((petit rire)) }\end{array}$ & $\begin{array}{l}\text { Négative } \\
\text { Il se sent } \\
\text { “insulté". } \\
\text { Il ressent } \\
\text { movirì comme } \\
\text { une } \\
\text { provocation. }\end{array}$ & $\begin{array}{l}\text { Il s'adresse à un } \\
\text { autre client. }\end{array}$ & $/ / / /$ \\
\hline
\end{tabular}




\begin{tabular}{|c|c|c|c|c|c|}
\hline 5 & $\begin{array}{l}\text { Sérieuse, } \\
\text { ni provocation, } \\
\text { ni jeu. } \\
\text { Insistance. }\end{array}$ & $\begin{array}{l}(\mathrm{t} 18) \text { Me li dai }((2 \mathrm{~s})) \text { i } \\
\text { caffé me li dai ? ((4 s)) } \\
\text { Dai muoviti! }\end{array}$ & $\begin{array}{l}\text { Neutre } \\
\text { Muoviti est } \\
\text { neutre, ne } \\
\text { représente } \\
\text { pas un objet } \\
\text { de conflit } \\
\text { ultérieur. }\end{array}$ & $\begin{array}{l}\text { Ale insiste, il } \\
\text { force ch à } \\
\text { s'excuser, mais } \\
\text { ne donne pas } \\
\text { d'importance } \\
\text { particulière à } \\
\text { cette } \\
\text { occurrence : il se } \\
\text { concentre sur le } \\
\text { movirì précédent. }\end{array}$ & $\begin{array}{l}\text { (t19) Chiedimi } \Sigma \\
\text { cusa! }\end{array}$ \\
\hline
\end{tabular}

Une première interprétation de la réaction d'Ale nous amènerait à considérer celle-ci comme une variable dépendante de l'usage du sarde plutôt que de l'italien. Toutefois, l'attitude négative d'Ale envers l'occurrence sarde «movirì » ne peut se généraliser à la langue sarde en tant que telle, d'autant plus que cette conversation a été enregistrée dans un quartier de Cagliari où la communication en sarde est courante. Ainsi, une interprétation de la réaction négative à « movirì » basée sur un jugement a priori négatif du sarde et motivée par une simple équation « utilisation du sarde = propos à rejeter » ne nous paraît pas satisfaisante.

L'attitude négative vers la langue locale est motivée plutôt par l'attribution de valeurs relatives au sarde et à l'italien relevant de leur opposition dans ce contexte d'usage particulier, où l'alternance des deux codes endosse une fonction d'indice de deux comportements divergents. Les réactions de la part d'Ale à cet élément lexical seraient ainsi à interpréter comme le résultat de cette situation interactionnelle spécifique, dans laquelle l'emploi du sarde est d'abord associé à un ordre et à une provocation par Ch qui met en danger sa face - au sens goffmanien - dans un contexte d'interaction publique. Parallèlement, quand Ch utilise l'italien «muoviti » au lieu du sarde "movirì», il vise notamment à éviter la cause du conflit identifiée avec l'élément lexical «movirì » sans pourtant vouloir se rendre totalement à la démonstration de « force » d'Ale.

Certains éléments caractérisent aussi bien la conversation entre Ale et Ch que celles qui se sont déroulées dans le bureau de l'ASL, bien que tant le contexte large que les profils des participants et leurs comportements divergent considérablement.

En effet, dans ces conversations asymétriques, un des interactants essaie de gérer la situation communicative et, dans une certaine mesure, peut le faire grâce à une autorité qui lui dérive de son statut social et professionnel ou, tout simplement, d'une personnalité plus forte face à son interlocuteur. Cette condition lui permet de choisir et imposer un certain niveau expressif qui caractérise l'échange verbal. La commutation codique, même lorsqu'elle est relative à un seul énoncé - voire, un seul mot - peut constituer un indicateur significatif de ce rapport entre les participants.

Ainsi, dans le bureau de l'ASL, l'employé utilise le sarde pour plaisanter avec F26 à laquelle il donne des renseignements. Il peut même se moquer d'elle avec un usage marqué et explicite du français et également du sarde dans la clôture de l'interaction. La présence d'une autre adulte, la mère de la jeune fille, n'empêche pas cet abaissement de registre, qui est signalé (avec d'autres indices) par le code-switching. Au contraire, quand le commentaire en sarde est produit par G, le même employé utilise l'italien comme langue de l'interaction et ne s'oriente pas vers une convergence. Cependant, il utilise le 
sarde pour exprimer un commentaire avant de commuter de nouveau en italien. Autrement dit, dans ce contexte fondamentalement formel et asymétrique, l'emploi du sarde de la part de $G$ est perçu comme inapproprié à la situation communicative. En revanche, le plurilinguisme semble plus «légitime » à l'employé, qui gère les choix codiques comme des moyens lui assurant le contrôle des paramètres de la situation énonciative.

Dans le cas de l'opposition «movirì - muoviti » il n'y a pas de véritable négociation concernant le code : l'italien n'est pas en concurrence avec le sarde comme langue de l'interaction et l'utilisation de l'élément sarde a plutôt une fonction régulatrice de la clé discursive. Plusieurs éléments sont à la base de l'interprétation négative de "movirì ", effectuée par Ale. Premièrement, $\mathrm{Ch}$ adopte un ton insolent qui viole la position publique d'Ale. Deuxièmement, l'injonction en sarde a été réalisée devant les autres clients d'Ale, mettant en cause sa légitimité dans la sphère professionnelle. Pour ces raisons, Ale réagit en imposant son autorité, met un interdit à l'usage du sarde, coïncidant à une menace à son image publique. Nous sommes confronté à la manifestation d'une valeur émotive forte accordée aux usages disponibles dans les répertoires verbaux. Dans le cadre de cette interaction, Ale, qui est en position de "force" par rapport à $\mathrm{Ch}$, contrôle les choix codiques mis à l'œuvre dans l'activité langagière. Ainsi, il impose et régule la production plurilingue, selon le sens et les fonctions pragmatiques qu'il lui confère.

\section{Conclusion}

L'objectif de cet article était de contribuer à la réflexion sur le rôle du contexte dans un cadre de production bilingue à travers la présentation d'interactions enregistrées dans un milieu urbain. Nous avons essayé de mettre en relief le lien existant entre le codeswitching et l'organisation conversationnelle, selon un point de vue théorique où l'événement communicatif est évalué en tant que tour de parole inséré dans un contexte discursif spécifique comprenant tous les éléments de l'échange verbal. La démarche interactionnelle permet de saisir la valeur communicationnelle du code-switching, lequel se configure comme un indice de contextualisation contribuant à une réinterprétation constante de l'échange et de l'activité de négociation que chacun des interactants met constamment en place lors de l'interaction.

Dans une perspective liée au cadre sociolinguistique du terrain d'enquête choisi, l'analyse des pratiques interactionnelles spontanées nous amène à souligner l'utilité d'observer les manifestations du contact dans une dimension dynamique. Notamment, dans l'aire italoromane, cette analyse laisse émerger la présence d'un continuum interne au répertoire verbal de la communauté linguistique considérée et permet de mettre l'accent sur les phénomènes de convergence affectant aussi bien les usages que les structures de la variété locale et de la langue de prestige. Dans un cadre d'actions d'aménagement linguistique, cette considération peut constituer un élément de réflexion important permettant de dépasser les limites constituées par le facteur identitaire "nationalitaire " qui est encore prééminent dans une conception strictement statique, diglossique du bilinguisme. Ainsi, le rapport sarde-italien, malgré le statut socio-historique et culturel spécifique du domaine linguistique sarde, peut être assimilé à celui caractérisant les autres variétés dialectales du domaine italo-roman donnant lieu à une situation de bilinguisme avec dilalie, se caractérisant par des emplois mixtes et par l'utilisation des deux langues dans des domaines a priori inattendus (Berruto 1993). 
contexte s'affirme ainsi comme une entité à plusieurs dimensions. La prise en compte de cet éventail de dimensions nous paraît favoriser une interprétation plus riche et " cohérente » des phénomènes linguistiques dans l'évènement conversationnel et, dans le cas spécifique du rapport se développant entre langues diverses en situation de contact, cette attention peut contribuer à éclaircir davantage le champ de l'analyse du répertoire langagier d'une communauté bi-plurilingue.

\section{BIBLIOGRAPHIE}

Alfonzetti G. (1992), Il discorso bilingue. Italiano e dialetto a Catania, Milano, F. Angeli.

Álvarez Cáccamo C. (1990), "Rethinking conversational code-switching: Codes, speech varieties, and contextualization", in Proceedings of the 16th Annual Meeting of the Berkeley Linguistics Society, Berkeley, BLS: 3-16.

Álvarez Cáccamo C. (2000), "Para um modelo do "code-switching" e a alternância de variedades como fenómenos distintos : dados do discurso galego-português/espanhol na Galiza", Estudios de Sociolinguïstica $1: 111-128$.

Artizzu L. (1997), Il dizionario di Cagliari, Cagliari, Della Torre.

Auer P. (1984), Bilingual conversation, Amsterdam-Philadelphia: John Benjamins.

Auer P. (1992), Introduction: John Gumperz' Approach to Contextualization, in Auer, Di Luzio (éds): 1-37.

Auer P., Di Luzio A. (éds) (1992), The contextualization of language, Benjamins.

Auer P. (1995), The pragmatics of code-switching: a sequential approach, in Milroy L., Muysken P. (éds.), One speaker, two languages. Cross-disciplinary perspectives in code-switching, Cambridge, CUP: $115-35$.

Auer P. (éd.) (1998), Code-switching in conversation. Language, interaction and identity, London, Routledge.

Auer P. (1999), "From codeswitching via language mixing to fused lects: Toward a dynamic typology of bilingual speech", International Journal of Bilingualism 3/4: 309-332.

Auger N. (2001), "'Claro qué y'a du miel'. Mélange de langues chez des hispanophones en situation professionnelle en France", in Canut C., Caubet D. (éds), Comment les langues se mélangent. Codeswitching en Francophonie, Paris, L'Harmattan : 73-84.

Beguelin M.-J. (éd.) (2000), De la phrase aux énoncés : grammaire scolaire et descriptions linguistiques, Bruxelles, De Boeck - Duculot.

Berruto G. (1990), "Italiano regionale, commutazione di codice e enunciati mistilingui", in Cortelazzo M. A., Mioni A. M. (éds), L'italiano regionale. Atti 180 Congresso S.L.I. (Padova Vicenza, 14-16/09/1984), Roma, Bulzoni : 105-130.

Berruto G. (1993), "Varietà del repertorio", in Sobrero A. A. (éd.), L'italiano contemporaneo. La variazione e gli usi, Roma-Bari, Laterza: 3-36.

Corela, HS-1 1 | 2012 
Berruto G. (2005), "Dialect / standard convergence, mixing, and models of language contact: the case of Italy", in Auer P., Hinskens F., Kerswill, P. (éds.), Dialect change. Convergence and divergence in European Languages, Cambridge, CUP : 81-95.

Cerruti M. (2004), "Aspetti pragmatico-funzionali della commutazione di codice italiano dialetto : un'indagine a Torino". Vox Romanica 63 : 94-127.

Cerruti M. et Regis R. (2005), '"Code-switching" e teoria linguistica : la situazione italo-romanza", Italian Journal of Linguistics $17: 179-208$.

D’Agostino M. (1996), "Spazio, città, lingue. Ragionando su Palermo", RID 20 : 35-87.

Depau G. (sous presse), "Italiano e dialetto nell'area urbana di Cagliari (Italia)", in Actes du CILPR - XXV (Innsbruck, 03-08/09/2007).

Depau G. (2005), "Considerazioni sulla scrittura esposta" RID 29 : 149-167.

Depau G. (2009a), "Contact de langues dans le domaine italo-roman : exemples de convergence linguistique Sarde - Italien dans l'aire urbaine de Cagliari", in Alén Garabato C., Arnavielle T., Camps C. (éds), La Romanistique dans tous ses états, Paris, L'Harmattan : 139-158.

Depau G. (2009b), "Les graffitis comme image du rapport italien - dialecte chez les jeunes dans le contexte urbaine à Cagliari (Sardaigne)", in Dalbera-Stefanaggi M.-J., Simoni-Aurembou M.-R. (éds.), Images de la langue : représentations spatiales, sémantiques et graphiques (édition électronique) Actes du 132e Congrès du CTHS "Images et imagerie" (Arles, 16-21/04/2007) : 108-127.

Depau G. (2010), "Variation linguistique en milieu urbain", in Horiot B. (éd.), La dialectologie d'hier et d'aujourd'hui (1906-2006), Actes du colloque de Lyon (7-9/12/2006), Lyon, Centre d'études linguistiques J. Goudet : 411-424.

Dettori A. (2002). "La Sardegna", In M. Cortelazzo, C. Marcato, N. de Blasi, G. P. Clivio (éds.), I dialetti italiani, Torino, Utet : 898-959.

Di Meglio A. (2007), "Langue(s), identité(s) et scolarité : la question du rapport $\ddagger$ l'autre et de la citoyenneté à partir de l'exemple corse", in Burban C., Lagarde C. (éds), L'école, instrument de sauvetage des langues menacées?, Perpignan : PUP : 123-135.

Dittmar N. (2005), "Sociolinguistique et analyse du discours : quel type d'interface ?" Langage et Société $114:$ 48-71.

Duranti A., Goodwin Ch. (éds.) (1992), Rethinking context, Cambridge, CUP.

Giles H., Coupland J., Coupland N. (éds.) Contexts of accomodation. Developments in applied sociolinguistics, Paris-Cambridge, MSH - CUP: 1-68.

Goffman E. (1981), Forms of talk, Philadelphia: University of Pennsylvania Press.

Gumperz J. J. (1982), Discourse strategies, Cambridge, CUP.

Gumperz J. J. (1992), "Contextualization revisited", in Auer-Di Luzio (éds) : 39-54.

Lüdi G., Py B. (1986), Être bilingue, Berne, Peter Lang.

Kerbrat-Orecchioni C. (2005), Le discours en interaction, Paris, Colin.

Kerbrat-Orecchioni C. (2006), Les actes de langage dans le discours, Paris, Colin.

Kleiber G. (1999), Il y a contexte et contexte, in Plénat M. et alii (éds), L'emprise du sens.

Structures linguistique et interprétations, Amsterdam/Atlanta, Rodopi : 167-181.

Maître R. (2003), "La Suisse romande dilalique", Vox Romanica 62 : 170-181. 
Micheli R. (2006), "Contexte et contextualisation en analyse du discours : regard sur les travaux de T. Van Dijk" Semen 21, Catégories pour l'analyse du discours politique [en ligne], URL : http:// semen.revues.org/document1971.html.

Mondada L. (2006), "La question du contexte en ethnométhodologie et en analyse conversationnelle", Verbum XXVIII, 2-3 : 111-151.

Mondada L. (2007), "Le code-switching comme ressource pour l'organisation de la parole-eninteraction", Journal of language contact $1: 168-197$.

Paveau M.-A. (2007), "Discours et cognition : les prédiscours entre cadres internes et environnement extérieur" CoReLa no spécial, Cognition, discours, contextes [en ligne], l'URL : http://edel.univ-poitiers.fr/corela/document.php?id=1672.

Paulis G. (1997), "Prefazione", in Artizzu (1997) : 7-9.

Porru V. R. (1832), Nou dizionariu universali sardu-italianu, Cagliari, Tipografia Arciobispali.

Rindler Schjerve R. (1998), "Codeswitching as an indicator for language shift ? Evidence from Sardinian-Italian bilingualism", in Jacobson R. (éd.), Codeswitching worldwide, Mouton de Gruyter, Berlin-New York: 221-247.

Sacks H., Schegloff E. A., Jefferson G. (1974), “A simplest systematics for the organization of turntaking for conversation”, Language 50: 696-735.

Schegloff E. A., Sacks H. (1973), “Opening up closings”, Semiotica $8: 289-327$.

Sebba M., Wootton T. (1998), “We, They and Identity: Sequential vs. Identity-related explanation in code-switching", in Auer P. (éd.): 262-289.

Spano G. (1851), Vocabolario Sardo - Italiano e Italiano - Sardo, Cagliari, Tipografia nazionale.

Wagner M. L. (1960-64) Dizionario Etimologico Sardo, Heidelberg, Winter.

\section{NOTES}

1. Micheli (2006: Introduction). Parmi les études portant sur la notion du contexte en linguistique, cf. Auer (1992), Kerbrat Orecchioni (1996), Colletta, de Nuchèze (2002), Mondada (2006), Paveau (2007). Tous ces auteurs s'intéressent notamment - suivant différentes démarches - à l'analyse de discours (en sens très général), dont notre perspective d'étude se rapproche.

2. Ces productions se situent dans une échelle de formalité organisée sur les deux pôles [+ formel] (situations de conversation asymétrique comme, par exemple, entre fonctionnaire publique et usager) et, respectivement, [+ informel] (conversations symétriques comme, par exemple, entre étudiants devant leur lycée). Ces degrés indiquent, bien sûr, des tendances se référant à de situations conversationnelles bien plus complexes: en effet, dans plusieurs situations «+ formelles» des rapprochements de registre se produisent entre les interlocuteurs, tandis que dans les situations «+ informelles" les moments de distanciation ne sont pas rares. Cf. Depau (2010).

3. La Sardaigne est une région à statut spécial au sein de la République italienne; le Conseil régional sarde jouit d'une souveraineté à l'intérieur de son territoire, telle que le pouvoir d'adopter des lois sur des sujets spécifiques - par exemple sur le patrimoine culturel. L'île compte environ 1,650,000 habitants, dont environ 1,300,000 locuteurs de Sarde. La Loi Nationale 482/1999 et la Loi Régionale 26/1997 visent à la sauvegarde et valorisation de la langue sarde, dont traditionnellement les linguistes mettent en relief les spécificités structurelles vis-à-vis des autres langues romanes et notamment sa proximité de la source latine. L'adoption officielle, en 
2006, d'une variété standard de sarde (à côté de l'italien, qui garde sa priorité institutionnelle) pour les documents officiels de l'administration régionale, rentre dans le cadre des actions prévues par la Loi Régionale 26/1997.

4. Au niveau lexical, cette italianisation était déjà claire pour les lexicographes du $19^{\mathrm{e}}$ siècle (en particulier Porru 1832 ; Spano 1851) qui constatent l'importance des contacts avec les Piémontais dès le $18^{\mathrm{e}}$ siècle. D'après Dettori (2002: 931), la présence de l'italien a déterminé d'une part l'italianisation du dialecte avec d'importantes modifications au niveau morphologique et syntaxique, d'autre part les situations d'emploi alternatif d'italien et sarde. Paulis (1997: 8), souligne le faible intérêt consacré par les linguistes au dialecte de Cagliari, considéré certes un dialecte sarde mais aussi expression d'une culture urbaine moins sarde que celle des centres ruraux de l'île ; cf. aussi Artizzu (1997 : 11-12).

5. Sur la distinction entre commutazione di codice (correspondant grosso modo à code-switching ou encore, code-switching interphrastique) et enunciazione mistilingue (code-mixing, codeswitching intraphrastique) au sein du phénomène général du code-switching, cf. Berruto (1990) ; cf. aussi Auer (1999), utilisant des distinctions semblables.

6. Ce caractère est présent aussi dans des situations - en principe - formelles et, comme le souligne Auer (1992), les situations plus ritualisées ne sont pas exemptes non plus de modifications liées au contexte d'énonciation.

7. À ce propos, cf. encore Gumperz, (1982 : 75) ; cf. aussi Mondada (2007). Ces deux catégories doivent être considérées comme des catégories interprétatives employées en premier lieu par les locuteurs eux-mêmes (et par conséquent, par l'analyste), permettant de repérer les fonctions particulières du passage codique dans des contextes spécifiques.

8. Cf. Cerruti, Regis (2005: 180-181). Auer (1995: 120) mentionne plusieurs conversational loci où la code-switching est particulièrement fréquent. Le fait que la plupart de ces fonctions tende à se manifester dans des situations de contact très différentes les unes des autres montre qu'elles sont largement généralisables, quoiqu'elles puissent à chaque fois assumer une valeur quantitative et qualitative différente en fonction des diverses conditions sociolinguistiques et socioculturelles. Compte tenu de cette variabilité quantitative et qualitative, ces nombreuses fonctions ne forment pas un inventaire fermé et exhaustif, mais plutôt une liste ouverte s'appliquant à un ensemble défini de données et susceptible, donc, d'élargissements, de modifications et d'interprétations nouvelles selon le corpus de situations de contact pris en examen.

9. Le terme «stratégie » est calqué sur l'anglais strategy, contenant aussi l'acception sémantique d'«ajustement», absente du terme français. Le développement du concept a donc provoqué l'importation du terme dans la tradition de recherche francophone. Cette question terminologique a bien sûr aussi une valeur épistémologique car il peut y avoir confusion et on peut interpréter le comportement langagier des locuteurs comme une activité préétablie, ce qui serait en contradiction avec l'attribution que nous défendons, d'un rôle direct joué sur la pratique du code-switching par le contexte dans l'activité de chaque interactant, qui avance dans l'interaction au même rythme que ses interlocuteurs et produit plutôt des ajustements. Le terme "tactique » serait peut-être plus approprié que "stratégie», en transposant dans le domaine linguistique la relation sémantique que ces deux termes entretiennent dans d'autres domaines de savoirs (domaine militaire et historique, analyse économique et politique, etc.). Une discussion sur ce sujet nous éloignerait des objectifs de notre étude; nous nous bornons donc à signaler l'ambiguïté du terme "stratégie " dans ce domaine disciplinaire, et préconisons une discussion plus approfondie dans un cadre épistémologique plus approprié.

10. Suivant Gumperz (1982: 95), cet emploi est « associated with in- and out-group experiences ». Sur la complexité des rôles attribués aux codes à l'intérieur d'une communauté, avec la possible superposition et le chevauchement de fonctions entre we- et they-code, cf. Sebba et Wootton (1998). Dans le domaine italo-roman cf. Alfonzetti (1992) ; Berruto (1985) ; Cerruti (2004); Depau (2009a, 
sous presse). L'emploi des deux codes n'est pas, toutefois, totalement « innocent », et dans certains contextes plus spécifiques leur valeur identitaire peut émerger; cf. Depau (2005; 2009b) (production de graffitis en italien et sarde); Di Meglio (2007) (graffitis en Corse); cf. aussi D’Agostino (1996) sur l'emploi du dialecte sicilien en tant qu'expression possible d'une adhésion à l'organisation (ou simplement, une « mentalité ») mafieuse selon les représentations des habitants de Palerme.

11. Conventions de Transcription:

$\mathrm{xxx} \rightarrow$ italien $; \underline{\mathrm{xx}} \rightarrow$ italien régional / éléments communs à l'italien et au sarde ;

$x x x$ è sarde $; \mathbf{x} \mathbf{x} \mathbf{x} \rightarrow$ mise en relief d'un ou plusieurs éléments du texte ;

$(())$ è commentaire du transcripteur, pause (indiquée en seconds) ;

$((=>)) \rightarrow$ destinataire principal du tour $;[. ..] \rightarrow$ omission de tours de parole ;

$<\mathrm{INC}>$ è un ou plusieurs éléments (phonétiques ou lexicaux) incompréhensibles ;

$\mathrm{xxx} ? \rightarrow$ intonation d'interrogation ; x x $! \rightarrow$ intonation d'exclamation ;

$\mathrm{xxx}$ ? ! $\rightarrow$ intonation descendante/ascendante, "questions rhétoriques »;

$\mathrm{XXX} \rightarrow$ augmentation du ton de la voix ; $\mathrm{x} \mathrm{x} \mathrm{:} \mathrm{è} \mathrm{prolongation} \mathrm{vocalique} \mathrm{;}$

$\mathrm{x} \mathrm{x} \mathrm{x} \mathrm{/} \mathrm{è} \mathrm{interruption,} \mathrm{autocorrection;} \mathrm{«} \mathrm{x} \mathrm{x} \mathrm{x}$ » è discours directe rapporté ;

$\mathrm{x} \mathrm{x} \mathrm{x}^{\mathrm{xx}} \rightarrow$ prononciation évanescente d'un ou plusieurs éléments phoniques de l'énoncé motivée par le débit de réalisation rapide.

Le sarde: transcription simplifiée adaptée aux règles orthographiques de l'italien. Les seuls caractères API utilisés concernent des réalisations phonétiques spécifiques du sarde récurrentes dans les extraits :

$[\Sigma]$ : fricative prépalatale sourde; même prononciation que français champ

[Z] : fricative prépalatale sonore ; même prononciation que français jour

Le digraphe tz est utilisé pour indiquer la consonne affriquée alvéolaire sourde. Ce choix est motivé aussi par le fait que cet élément graphique est utilisé dans l'écriture en sarde. Ex : intz andusu.

Les phénomènes de sandhi sont rapportés à travers le graphème italien le plus proche phonétiquement, comme dans l'ex. piga gustu « prends ceci » (<custu).

Les interlocuteurs sont identifiés en relation au sexe et à l'âge indicatif : par ex. «F45 » indique "personne de sexe Féminin âgée d'environ 45 ans ».

Traductions en français: nous avons opté pour une traduction pragmatique du contenu des extraits.

12. Notons que durant toute l'interaction, même lorsqu'il adopte cette attitude moqueuse vis-àvis de ses interlocutrices, EH50 s'adresse à elles à travers la forme de politesse (t8 : " glielo sto dicendo [...] glielo dico [...] se vuole $»)$.

13. Il s'agit de Mme Nerina Dirindin, qui était à l'époque adjoint au Président de la Région, chargée à l'hygiène, à la santé et à l'assistance sociale du conseil régional de la Région Sardaigne. Avec « il nostro illuminato » l'employé fait référence, probablement, à Renato Soru, ancien leader de la coalition de centre-gauche et qui, en qualité de Président de la Région Sardaigne, avait nommé Dirindin pour cette fonction institutionnelle.

14. «Mo' « n'est pas exclusif de l'aire sarde; il est présent aussi dans d'autres variétés (méridionales) de l'espace italo-roman.

15. Le double «allez » (" da' ajò ») adressé par Ch à son interlocuteur est le même du t6: il y a, ainsi, une certaine symétrie dans ses réactions au long de l'échange. «da' " est une forme de l'italien régional / italien informel, tandis que « ajò » est une forme sarde pénétrée dans l'italien régional de Sardaigne. 


\section{RÉSUMÉS}

Cet article vise à fournir des éléments de réflexion sur le rôle du contexte dans un cadre de production bilingue à travers la présentation d'interactions enregistrées dans un milieu urbain. La Sardaigne constitue notre domaine d'enquête spécifique. Cette étude se fonde sur l'analyse d'un corpus oral consistant d'un ensemble de textes bilingues « sarde-italien » recueillis (pour un total d'environ 16 heures d'enregistrements) dans des contextes interactionnels assez variés et se caractérisant par divers degrés de formalité. L'analyse effectuée dans notre étude est centrée principalement sur une démarche interprétative visant à mettre en relief la valeur communicationnelle des phénomènes de code-switching.

This article aims to provide evidence of the role of context in bilingual production, through the presentation of interactions recorded in an urban milieu. Sardinia is our specific research field. This study is based on the analysis of an oral 16-hour corpus consisting of "Sardinian-Italian" bilingual texts gathered in various interactional contexts and characterized by different degrees of formality. The analysis made in our study mainly focuses on an interpretative approach aiming to highlight the conversational value of code-switching phenomena.

INDEX

Keywords : code-switching, contextualisation, interaction, Italian, Sardinian

Mots-clés : code-switching, contextualisation, interaction, Italien, Sarde

\section{AUTEUR}

\section{GIOVANNI DEPAU}

Université Stendhal - Grenoble 3 / Université Sophia-Antipolis, Nice 